\title{
Mental body rotation with egocentric and object-based transformations in different postures: sitting vs. standing
}

\section{KIRSTEN BUDDE¹ | JOSÉ A. BARELA² | GABRIELLA A. FIGUEIREDO² | MATTHIAS WEIGELT¹}

1 Department of Sport and Health, Psychology and Movement Science, University of Paderborn, Germany.

2 Department of Physical Education, Institute of Biosciences, São Paulo State University, Rio Claro, SP, Brazil.

Correspondence to: Kirsten Budde, Department of Sport and Health, Psychology and Movement Science, University of Paderborn, Warburger Str. 100, 33102 Paderborn,

Germany.

email: kirsten.budde@uni-paderborn.de

https://doi.org/10.20338/bjmb.v14i2.165

\section{HIGHLIGHTS}

- Answers are faster in the egocentric compared to the object-based transformation.

- The RT rises with increasing rotation angle.

- Increasing rotation angle has a greater impact

on the object-based transformation.

- Participants commit more mistakes in the object-based transformation.

- There is no main effect or interaction for the factor posture.

\section{ABBREVIATIONS}

A1 object-based transformation task and standing posture

A2 object-based transformation task and sitting posture

B1 egocentric transformation task and standing posture

B2 egocentric transformation task and sitting posture

MBRT mental body-rotation task

MRT mental rotation task

RE response error

RT response time
BACKGROUND: A detail of previous studies on mental rotation, which has not received any attention so far, relates to the testing situation of the participants. In nearly every study, participants were tested in a sitting posture (and not standing). However, when considering embodied cognition approaches on mental processes, participants may not be able to fully exploit these processes when performing mental rotation tasks in a sitting posture.

AIM: Therefore, the aim of the present study is to examine the potential influence of two different postures (sitting vs. standing), when solving mental body rotation tasks.

METHOD: Sixteen participants (6 females) were tested in two mental body-rotation tasks (MBRT), requiring either an object-based spatial transformation (based on a same-different judgment) or an egocentric transformation (based on a left-right judgment) in a sitting and in a standing posture. Reaction times and response errors were analyzed in two three-way ANOVAs, with the factors orientation, task, and posture.

RESULTS: Results revealed an effect of orientation and task, indicating that participants performed better for egocentric than for object-based transformations. However, there was no effect of posture.

CONCLUSION: The different dynamics of postural control during sitting and standing do not induce different embodiment effects on mental rotation.

KEYWORDS: Mental rotation | Posture | Embodiment

\section{INTRODUCTION}

The mental rotation task (MRT) by Shepard and Metzler ${ }^{1}$ is a widely used paradigm to test people's visual spatial abilities in cognitive psychology. According to Steggemann-Weinrich and Weigelt, ${ }^{2}$ mental rotation skills signify people's "ability to spatially transform two-dimensional or three-dimensional objects or bodies from one orientation in mental space to another" (p. 173). In the standard MRT, two pictures of three-dimensional objects are shown side-by-side, whereupon one picture serves as a reference image and is therefore presented in an upright position and the other picture is displayed at various orientations. Participants' task is to decide if the two pictures depict the same or different objects (i.e., same-different judgment), regardless of the differences in orientation. ${ }^{1}$ Besides this classical MRT, previous studies have used a variety of different stimuli to examine the mental rotation skills using the psychometric testing 
approach. These included two-dimensional shapes, ${ }^{5}$ letters, ${ }^{6}$ images of human body parts, ${ }^{7}$ or whole human bodies. ${ }^{8}$ The present study investigates the mental rotation of ${ }^{1}$ human bodies in an extension of the standard MRT by Shepard and Metzler, 1 which is called the mental body-rotation task (MBRT). ${ }^{9}$

In general, three types of transformations can be distinguished based on an environmental reference frame, an egocentric reference frame, and an object-based reference frame. ${ }^{10,11}$ The environmental reference frame is defined relative to a fixed point of the environment, locating things relative to axes with respect to a fixed space. In contrast, the egocentric reference frame is defined relative to the self. Humans use this egocentric reference frame with the axes up-down, front-back, and left-right. The objectbased reference frame is the third type of spatial reference frames, which is defined relative to external objects. The object-based reference frame can be used either for characterizing the relationship between the parts of an object independent of the object's location in the environment or to locate an object relative to another object. ${ }^{10}$

A detail of most all previous studies on mental rotation, which has not received any attention so far, relates to the testing situation of the participants. That is, participants were always tested in a sitting posture (and not standing; but see Kaltner ${ }^{12}$ ). This certainly reflects the "natural" test scenario of laboratory research. However, when considering embodied cognition approaches on mental processes, $13,14,15$ it could be argued that participants may not be able to fully exploit these processes when performing mental tasks in a sitting posture. The theoretical framework of embodied cognition states a strong link between motor and mental processes, meaning that cognitive processes are deeply rooted in the body's interaction with the environment. ${ }^{15}$ There are two kinds of embodiment, which can explain the performance of spatial transformation: The first is spatial embodiment and assumes a bodily projection of the own body axe onto the embodied object, such as for the stimulus material in a MBRT. The second is motoric embodiment and suggests that the processes of imagining, observing, and executing actions all share the same motor representations ${ }^{13}$ In addition, Schütz-Bosbach and Prinz ${ }^{16}$ distinguish between offline and online effects in terms of embodied cognition. Online effects refer to the phenomenon, that ongoing actions influence the perception of similar or different actions. Offline effects are based on past movement experiences, which are stored in motor representations. These motor representations influence perception and decision making, even when a person is not moving. ${ }^{16}$

Interestingly object-based and egocentric transformations differ in the amount of embodiment. While object-based transformations rely on object-centered representations, egocentric (perspective) transformations rely on simulated movements of the own body, where proprioceptive information is more relevant. ${ }^{10}$ Therefore, for object-based spatial transformations (based on same-different judgments), it may not make a difference whether participants are sitting or standing. For egocentric transformations (based on leftright judgments), however, the posture in which participants perform may well influence their performance, especially, when they must rotate human figures, because under these

\footnotetext{
${ }^{1}$ Besides the computer-based approach (psychometric testing approach), there is also the chronometric approach to test for mental rotation abilities. Methodologically, it is based on a paper-and-pencil version of the mental rotation test (MRT), developed by Vandenberg \& Kuse, ${ }^{3}$ and has been used in many different versions (for a meta-analysis see Voyer ${ }^{4}$. The chronometric approach, however, is not within the focus of the present study and will therefore not be further addressed.
} 
conditions, participants most likely solve the task by drawing on own embodied representations of left and right.9,11

There are experimental observations from two different research areas, which suggest that the posture in which participants solve other perceptual-cognitive tasks, affects performance. The first observation comes from research on perceptual learning and was made rather by coincidence. Here, Faubert and Sidebottom ${ }^{17}$ examined high-level athletes from ice hockey, rugby, and soccer in a multiple-object tracking-task over an extended period of training (over 30 training sessions). These athletes were all sitting during the acquisition of the task (i.e. tracking multiple objects) and did not differ in the level of performance at the end of the study (as signified by similar learning curves). However, when they collected additional data from another ice hockey team, which was standing during the complete training period, they found that these athletes performed worse (as signified by flatter learning curves) than the athletes, who were sitting before. Faubert and Sidebottom ${ }^{17}$ attributed this surprising result to differences in motor load between the two posture conditions (sitting vs. standing) and stated that this "clearly demonstrates the link between balance control mechanisms and perceptual-cognitive demands" (p. 95).

The second observation comes from research by Bray and colleagues, ${ }^{18}$ who tested the link between different body positions and performance in a (subjective) visual judgment task. In their study, participants were asked to align a tilted rod to the earth's vertical. To give misleading cues to verticality, a rectangular frame surrounded the rod. In each trial, the experimenter tilted the frame to left or right and/or set the rod to left or right at a random angle of $25^{\circ}-35^{\circ}$ from the earth's vertical. Thereupon, participants had to adjust the rod to the earth's vertical. Importantly, they were tested under three different posture conditions: sitting on a chair, standing "at ease", and standing on a beam "balancing". As the results revealed, participants set the rod more accurately to the vertical line while they were balancing on the beam, as compared to the standing "at ease" and the sitting condition. The authors stated that this "suggests that information from the dynamics of balance improves the perception of orientation" (p. 609)..$^{18}$

The aim of the present study was to investigate the influence of embodied processes on the mental rotation of human bodies. With regards to previous studies, the following predictions were made: First, it is expected that participants perform generally better in the MBRT for egocentric transformations (as compared to the performance for object-based transformations). ${ }^{9}$ This should be reflected in the faster mental rotation times (as signified by a shallow RT-slope over different rotation angles) of human bodies, when the task requires a left-right judgment, and slower mental rotation times (as signified by a steeper RT-slope over different rotation angles), when the task requires a same-different judgment. Second, mental rotation performance should be influenced by the body posture in which participants solve the task. ${ }^{17,18}$ The different results of the two previous studies can be explained by task differences implying different task dynamics, as the dynamics of a tracking task ${ }^{17}$ is quite different than the perception of vertical lines/rods. ${ }^{18}$ Arguably, the results of Bray and colleagues ${ }^{18}$ are somewhat more relevant to the present study, because of the nature of the MBRT (i.e., visual-spatial alignment of human bodies). 


\section{METHODS}

\section{Sample}

Sixteen volunteers ( 6 females; mean age $=23.5$ years, age range $18-36$ years) with normal or corrected-to-normal vision participated in this experiment. They characterized themselves as neurologically healthy. All participants were students at the University of Paderborn in Germany and German was their native language. They were not paid for their participation but received course credit. Before being tested, everyone gave his or her written informed consent. None of the participants took part in any mental rotation experiment prior to this study. The study was approved by the local ethics committee of the university and was carried out in accordance with the Helsinki Declaration of 1975 .

\section{Apparatus}

The experimental set-up can be seen in Figure 1. A projector (Optoma) presented the stimuli onto a wall in the laboratory by using the software "Presentation" (Version 20.2, Neurobehavioral Systems). Participants viewed the experimental stimuli either standing or sitting on a chair, 3 meters away from the wall. The stimuli appeared in a size of $100 \mathrm{~cm}$ in diameter on a black screen. Verbal responses were given with a microphone (Rhode) linked via an usb-port with the computer. The threshold value for the microphone to be activated was adjusted to $0.1 \%$ of the maximum sound recording level.
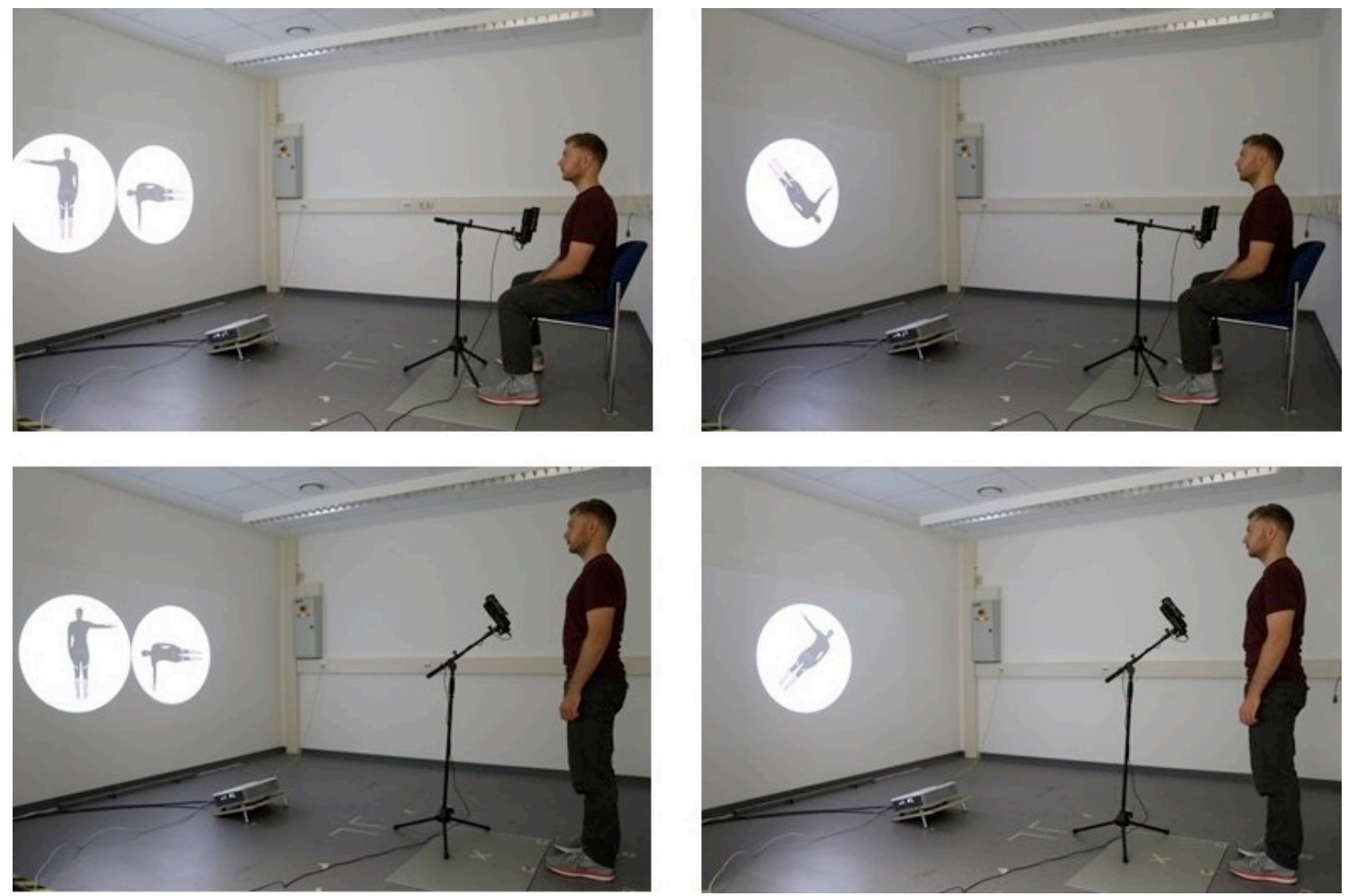

Figure 1. Experimental set-up under the different tasks and conditions. Note: Left panel, the task scenario for the same-different judgement is displayed for the sitting posture (picture on the top) and the standing posture (picture on the bottom). Right panel, the task scenario for the left-right judgement is depicted for the sitting posture (picture on the top) and the standing posture (picture on the bottom). 


\section{Stimulus Material}

Stimuli were taken from Steggemann et al. ${ }^{19}$. There were two different mental rotation tasks: One required an object-based spatial transformation and the other an egocentric perspective transformation (see Figure 2). In the object-based transformation, two images of a female person in back view perspective and with either the left arm or the right arm extended, were presented simultaneously on the screen. These images were either identical or mirror image reversals of each other. In each pair, the left image was arranged in an upright position $\left(0^{\circ}\right)$ and the orientation of the image at the right was rotated randomly in the picture plane (clockwise $0^{\circ}, 45^{\circ}, 90^{\circ}, 135^{\circ}, 180^{\circ}, 225^{\circ}, 270^{\circ}$, $315^{\circ}$ ), resulting in 32 different stimuli. Half of the trials presented pairs of identical objects and half displayed mirror-reversed objects, resulting in a same-different-judgment.

In the egocentric perspective transformation, a single image, depicting a female person with the left or the right arm outstretched, appeared on the screen. Therefore, a left-right decision was required. The person in the image was presented from back view and rotated randomly in the picture plane (clockwise $0^{\circ}, 45^{\circ}, 90^{\circ}, 135^{\circ}, 180^{\circ}, 225^{\circ}, 270^{\circ}$, $\left.315^{\circ}\right)$, resulting in 16 different stimuli.
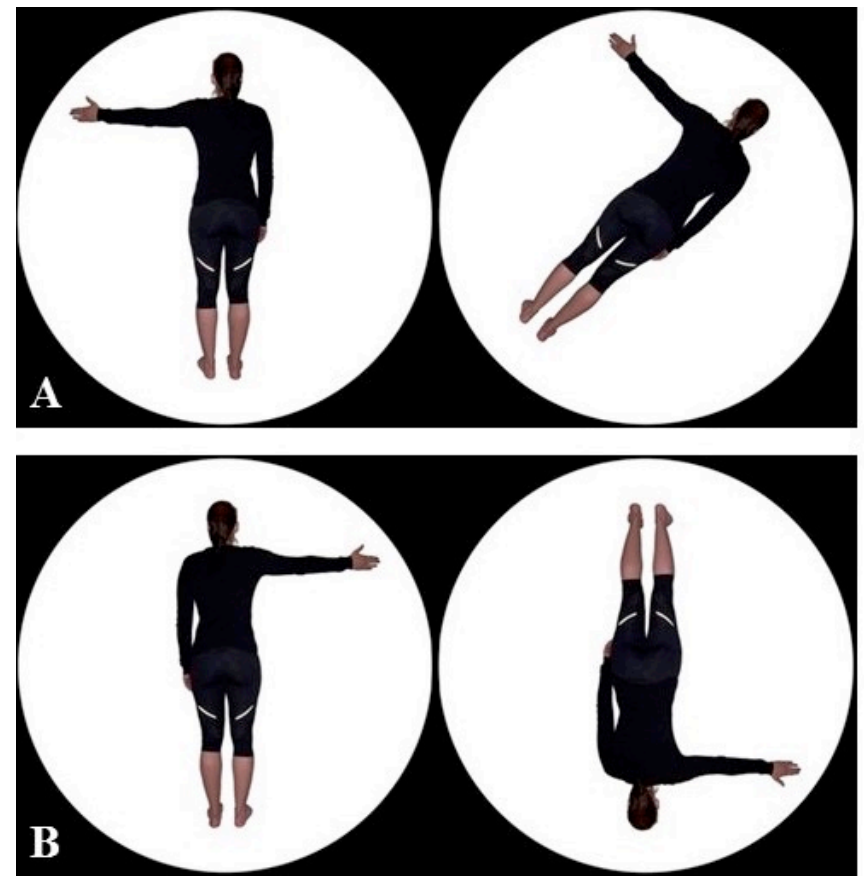
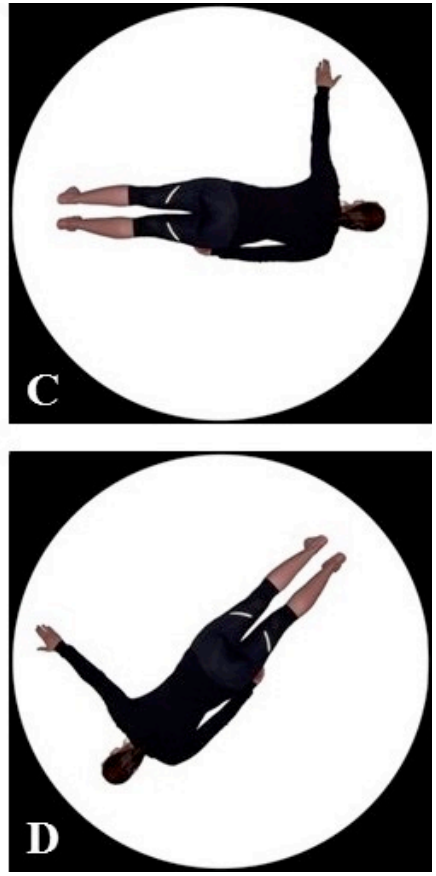

Figure 2. Examples of stimuli used in the experiment. Note: A) same-different judgment with $45^{\circ}$ angular disparity, same pictures; (B) same-different judgment with $180^{\circ}$ angular disparity, different pictures; (C) left-right judgment with $90^{\circ}$ angular disparity, left arm outstretched; (D) left-right judgment with $225^{\circ}$ angular disparity, right arm outstretched.

\section{Procedure and Task}

The test session lasted about 45 minutes and took place in the laboratory. Participants could read the standardized task introductions on their own. In the objectbased spatial transformation, participants had to decide as quickly and as accurate as possible if the presented stimulus on the right side was identical to the comparison stimulus on the left side. In the object-based transformation, participants had to answer 
"gleich" (German word for "same"), when the two stimuli were the same, and "ungleich" (German word for "different"), when the two stimuli were different. In the egocentric perspective transformation, only one picture of a woman in back-view perspective was presented. Participants were asked to determine as quickly and as accurate as possible whether the person raised her left arm or her right arm. They had to answer "links" (German word for "left"), when the left arm was raised, or to answer "rechts" (German word for "right"), when the right arm was raised. Participants were tested in four blocks: (A1) object-based transformation task and standing posture, (A2) object-based transformation task and sitting posture, (B1) egocentric transformation task and standing posture and (B2) egocentric transformation task and sitting posture. Half of the participants started with two blocks in the object-based transformation task (A1 and $\mathrm{A} 2$ ), while the other half started with two blocks in the egocentric transformation task (B1 and B2), before continuing in the other condition, respectively. Moreover, the order of the posture sitting vs. standing was balanced for the two tasks. The following four different orders of blocks were tested: (1) A1, A2, B1, B2; (2) A2, A1, B2, B1; (3) B1, B2, A1, A2; and (4) B2, B1, A2, A1.

Each trial started with a black screen. After $500 \mathrm{~ms}$, a white fixation cross appeared for $500 \mathrm{~ms}$, whereupon the stimuli were presented. The stimuli stayed on the screen until participants answered. In the case of a wrong answer, participants immediately received feedback and the German word "Fehler" appeared on the screen. Feedback was given for $1000 \mathrm{~ms}$.

To familiarize themselves with the stimuli and the tasks, participants performed two practice sessions: one with 32 test trials before the first block of the two blocks of the object-based transformation task and one with 16 test trials before the first of the two blocks of egocentric transformation task. The order of the trials within the practice session was randomized.

The entire experiment consisted of four test blocks (A1, A2, B1, B2) of 160 experimental trials in the two object-based transformation tasks (A1, A2) and 80 experimental trials in the two egocentric transformation tasks (B1, B2), resulting in 480 trials in total. In the object-based transformation task, each combination of the eight angular disparities of the right picture $\left(0^{\circ}, 45^{\circ}, 90^{\circ}, 135^{\circ}, 180^{\circ}, 225^{\circ}, 270^{\circ}, 315^{\circ}\right)$, the stimulus pairs (same or different), and the two images (original or mirrored) was presented five times in each test block. The 80 trials in the egocentric transformation task were composed of two stimulus types (person with left or right arm raised) $\mathrm{x}$ eight angular disparities $\left(0^{\circ}, 45^{\circ}, 90^{\circ}, 135^{\circ}, 180^{\circ}, 225^{\circ}, 270^{\circ}, 315^{\circ}\right) \times$ five repetitions of each combination. In the object-based transformation task, half of the trials showed the same and the other half showed different images. In the egocentric transformation task, half of the trials showed the person raising the left arm and half of the trials displayed the person raising the right arm. The order of the presentation of the stimuli was randomized. Between the blocks, participants could decide how long they wanted to have a break.

\section{Data analysis}

Data (response time and response error) were recorded by using the software "Presentation" and analyzed with two three-way ANOVAs, including the factors angular disparity $\left(0^{\circ}, 45^{\circ}, 90^{\circ}, 135^{\circ}, 180^{\circ}, 225^{\circ}, 270^{\circ}, 315^{\circ}\right)$, MR task (object-based vs. egocentric), and posture (standing vs. sitting), as independent variables, and response time (RT) and response error (RE), as dependent variables. RTs faster than $100 \mathrm{~ms}(0 \%)$ and slower than $1500 \mathrm{~ms}(2,34 \%)$ were defined as outliers and excluded from statistical 
Brazilian Journal of Motor Behavior

analysis, as well as data from incorrect trials (1,76 \%). As incorrect trials, we considered trials in which participants' answer was wrong. Correct (RT) and incorrect (RE) trials were analyzed separately. Data from the practice sessions were not analyzed. The Greenhouse-Geisser adjustment was used to correct for violations of sphericity and posthoc $t$-test were Bonferroni-Holm adjusted.

\section{RESULTS}

\section{Response Time}

The RT pattern for the two postures in the object-based spatial transformation (A) and in the egocentric perspective transformation $(B)$ can be seen from Figure 3 . The ANOVA displayed a main effect for MR task, $F(1,15)=154.664, p<.001, \eta^{2} p=.912$. Accordingly, participants were significantly faster in the egocentric transformation $(M=616$ $\mathrm{ms}, S D=69 \mathrm{~ms})$ than in the object-based transformation $(M=861 \mathrm{~ms}, S D=101 \mathrm{~ms})$. There was also a main effect of angular disparity, $F(1.363,20.440)=218.357, p<.001$, $\eta^{2} p=.936$, with the RT steadily increasing. Post-hoc $t$-test indicated that RT differed significantly from each angular disparity to the proximate one (all $p<.001)$. The interaction between condition and angular disparity reached significance $(F(1.650,24.755)=11.455$, $p=.001, \eta^{2} p=.433$ ), whereupon the increase of rotation angle had a greater impact on object-based transformation. Post-hoc t-test showed significant mean differences for all increases in angular disparity (all $p<.005)$, except the last one between $135^{\circ}$ and $180^{\circ}(p$ $=.052$ ). There was no main effect for posture and no significant two-way interaction, neither between condition and posture, nor between posture and angular disparity. Furthermore, the three-way interaction between angular disparity, stimulus condition and posture also failed to reach significance.

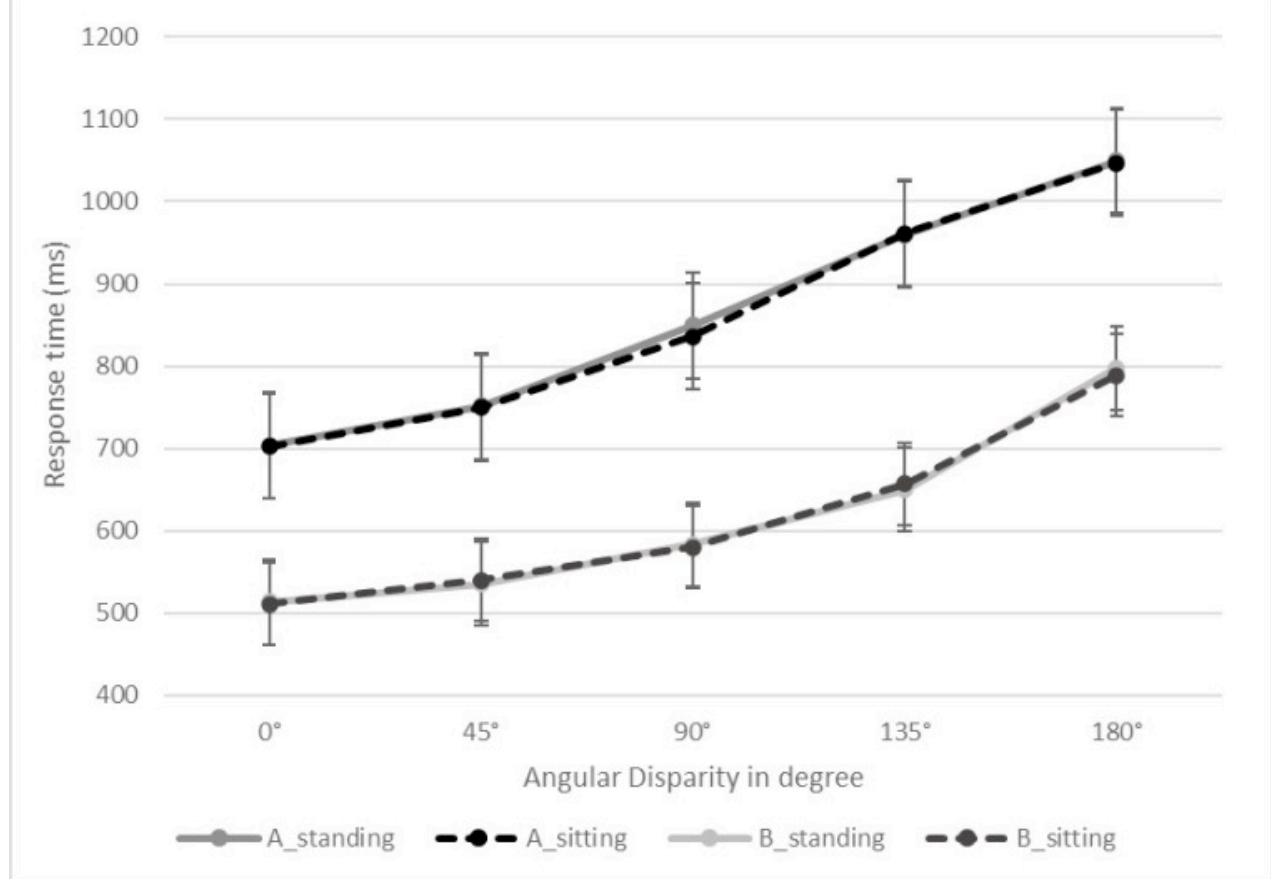

Figure 3. Response time (RT). Note: Note. Mean response times $(\mathrm{RT})$ in milliseconds $( \pm \mathrm{SE})$ for the objectbased transformation (A) and the egocentric transformation (B). 


\section{Response Error}

Figure 4 provides the RE pattern for both postures in the object-based spatial transformation $(A)$ and the egocentric perspective transformation (B). The ANOVA revealed a main effect of MR task $\left(F(1,15)=16.617, p=.001, \eta^{2} p=.526\right)$, showing that participants committed significantly more mistakes in the object-based transformation $(2.7 \%)$ than in the egocentric transformation $(0.3 \%)$. Also, a main effect for angular disparity $\left(F(1.847,27.710)=5.917, p=.008, \eta^{2} p=.283\right)$ was found. Post-hoc $t$-test revealed that $\mathrm{RE}$ differed significantly only between rotation angles of $90^{\circ}$ and $135^{\circ}(p$ $=.036)$. Moreover, there was a significant interaction between condition and angular disparity $\left(F(1.903,28.549)=4.533, p=.021, \eta^{2} p=.232\right)$. Post-hoc $t$-test showed only a significant mean difference between $90^{\circ}$ and $135^{\circ}(p=.044)$. There was no main effect for posture and no significant two-way interaction between condition and posture, as well as between posture and angular disparity. Furthermore, there was no three-way interaction between condition, posture, and angular disparity.

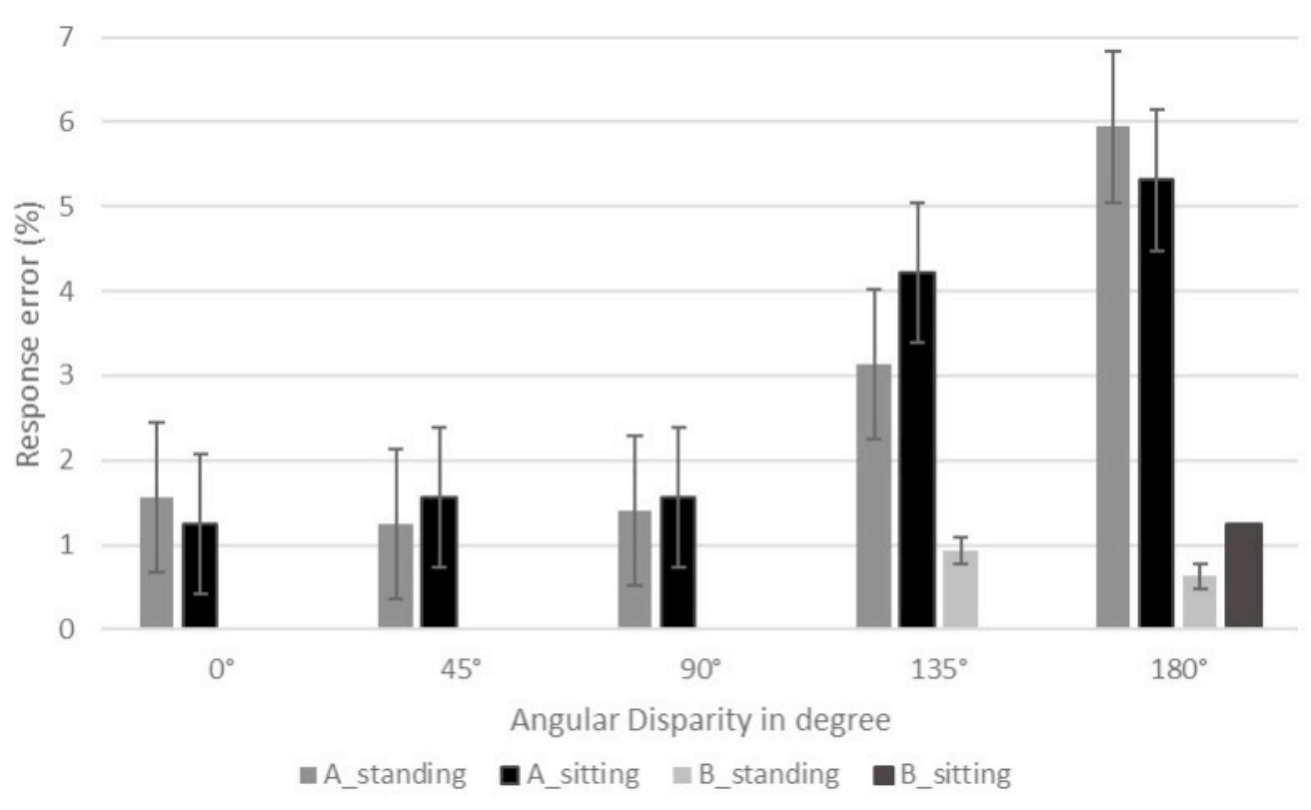

Figure 4. Response error $(\mathrm{RE})$. Note: Mean response errors $(\mathrm{RE})$ as percentages $( \pm \mathrm{SE})$ for the object-based transformation $(A)$ and the egocentric transformation $(B)$.

\section{DISCUSSION}

The aim of the present study was to investigate the influence of embodied processes on the mental rotation of human bodies. Therefore, for the first time, participants performed two MBRT in a sitting and in a standing position. In one MBRT, two pictures of a female person in back view perspective were presented and participants were asked for a same-different judgment, whereas in the other MBRT, only one picture of a female person in back view rising her left or right arm was displayed and the task required a left-right judgment. According to Zacks and colleagues ${ }^{11,20}$ and Jola and Mast, ${ }^{9}$ the first MBRT induced an object-based transformation and the second MBRT induced an egocentric transformation in the participants. 
In general, the results confirmed the predictions derived from previous studies on mental rotation of human bodies. ${ }^{9,21,22,23}$ That is, RTs and REs increase for visual-spatial transformations of human bodies, the more the stimuli have to be mentally rotated. In addition, participants performed better for egocentric transformations (i.e., requiring a leftright judgment) than for object-based transformations (i.e., requiring a same-different judgment), as reflected in faster response times and fewer errors. Thus, using a similar argumentation as other authors of previous studies,, 924 aligning oneself into the person displayed is faster than to spatially align and compare two objects.

Concerning the specific testing situation, it was expected that embodied processes would influence the mental rotation of human bodies, depending on the body posture in which participants solved the task (sitting vs. standing). ${ }^{17,18}$ One previous study on the perception of visual orientation demonstrated better performance when the balance of participants was challenged, ${ }^{18}$ whereas the opposite was observed during a study on perceptual training. ${ }^{17}$ In the present experiment, however, the two different postures examined did not lead to any effects on participants' mental rotation performance.

From an embodiment perspective, ${ }^{13,14,15}$ the latter result is surprising, because it can be assumed, that the two different body postures (sitting vs. standing) challenged the dynamics of balance control to different degrees. However, the challenge for the dynamics of balance control may not have been big enough, given the task dynamics in the present experiment. Besides sitting and standing at ease, Bray et al. ${ }^{18}$ had participants also perform while standing on a balance beam, which poses a greater challenge to the dynamics of balance control. This notion is supported by the observation, that the coupling between visual information and body sway is affected in (young and older) adults, when the basis of support is being manipulated. ${ }^{25}$ Thus, embodied processes may not have had a greater effect on participants' performance, because postural control processes (i.e., to keep static balance) did not interfere with perceptual-cognitive processes (i.e., to perform mental body rotations). Future studies should therefore examine participants' mental rotation performance under conditions in which their balance is challenged to a greater degree (e.g., on a balance beam).

It could be, however, that embodied processes influenced the mental rotation of human bodies, but were not detected in the present experiment. Arguably, the behavioural measures assessed (RT and RE) were not sensitive enough to show potential differences between the sitting and standing posture, although these measures are sufficient to demonstrate differences between egocentric transformations and object-based transformations. ${ }^{21,22} \mathrm{~A}$ more sensitive measure may be to examine the dynamics of balance control as postural sway on a force plate. In fact, a previous study by Stins and colleagues ${ }^{26}$ has demonstrated, that the semantic processing of sentences involving different daily activities of high, low or no physical effort effected participants' body sway in a selective way. In a future study, participants should be placed on a force plate when solving mental rotation tasks.

Concerning the embodiment approach 13,14,15 and the online and offline effects mentioned by Schütz-Bosbach and Prinz, ${ }^{16}$ processes of action and perception influence each other, because of similarity and contrast. Embodiment can help to encode and spatially represent rotated stimuli, as spatial embodiment moderates the mapping of one's own body into the presented stimulus, based on the knowledge of body structure, while motoric embodiment moderates the postural spatial configuration during the mental rotation process. Therefore, mental rotation processes and embodiment can hardly be 
separated. ${ }^{8}$ At the same time, there also seem to be limits to the embodiment argument, as in the present study, participants were similar able to map their own body representation to the stimulus figure while sitting or standing, suggesting that not all postural changes influence the performance in the MBRT.

\section{CONCLUSION}

For the first time, participants performed a MBRT in a sitting and standing position. It was of interest, if the different demands on balance control exhibited in these two postures would affect participants mental rotation performance. The present results did not show any differences between the two positions (sitting vs. standing, respectively), although previous studies have reported an influence of body posture on the perception of visual orientation ${ }^{18}$ and on perceptual training. ${ }^{17}$ Future studies should consider to test participants in more challenging body postures (for example, placing participants on a balance beam) and to use more sensitive measures (for example, a force plate to assess body sway), in order to further examine mental rotation performance from an embodiment perspective. ${ }^{13,14,15}$

\section{REFERENCES}

1. Shepard RN, Metzler J. Mental rotation of three-dimensional objects. Science. 1971;171: 701-703. doi: https://doi.org/10.1126/science.171.3972.701.

2. Steggemann-Weinrich $Y$, Weigelt M. Mental rotation skills. In: Hackfort D, Schinke RJ, Strauss B, eds. Dictionary of Sport Psychology - Sport, Exercise, and Performing Arts. London, UK: Academic Press; 2019:173.

3. Vandenberg SG, Kuse AR. Mental rotations, a group test of three-dimensional spatial visualization. Percept Mot Skills. 1978;47:599-604. doi: https://doi.org/10.2466/pms.1978.47.2.599.

4. Voyer D. Time limits and gender differences on paper-and-pencil tests of mental rotation: a meta-analysis. Psychon Bull Rev. 2011;18: 267-277. doi: https://doi.org/10.3758/s13423-010-0042-0.

5. Cooper LA. Mental rotation of random two-dimensional shapes. Cogn Psychol. 1975;7:2043. doi: https://doi.org/10.1016/0010-0285(75)90003-1.

6. Voyer D, Jansen P, Kaltner S. Mental rotation with egocentric and object-based transformations. Q J Exp Psychol. 2017;70:2319-2330.

doi: http://doi.org/10.1080/17470218.2016.1233571.

7. Bläsing $B$, Brugger $P$, Weigelt $M$, Schack $T$. Does thumb posture influence the mental rotation of hands? Neurosci Lett. 2013;534:139-144. doi: https://doi.org/10.1016/j.neulet.2012.11.034.

8. Amorim MA, Isableu B, Jarraya M. Embodied spatial transformations: "Body analogy" for the mental rotation of objects. J Exp Psychol Gen. 2006;135:327-347. doi: https://doi.org/10.1037/0096-3445.135.3.327. 
9. Jola C, Mast FW. Mental object rotation and egocentric body transformation: Two dissociable processes? Spatial Cognit Comput. 2005;5:217-237. doi: https://doi.org/10.1080/13875868.2005.9683804.

10. Zacks JM, Michelon P. Transformations of visuospatial images. Behav Cogn Neurosci Rev. 2005;4:96-118. doi: https://doi.org/10.1177/1534582305281085.

11. Zacks JM, Mires J, Tversky B, Hazeltine E. Mental spatial transformations of objects and perspective. Spatial Cognit Comput. 2002;2:315-332. doi: https://doi.org/10.1023/A:1015584100204.

12. Kaltner $S$, Jansen $P$, Riecke BE. Stimulus size matters: do life-sized stimuli induce stronger embodiment effects in mental rotation? Cogn Psychol. 2017;29:701-716. doi: https://doi.org/10.1080/20445911.2017.1310108.

13. Barsalou LW. Perceptual symbol systems. Behav Brain Sci. 1999;22:577-660. doi: https://doi.org/10.1017/S0140525X99002149.

14. Wexler M, Kosslyn SM, Berthoz A. Motor processes in mental rotation. Cognition. 1998;68:77-94. doi: https://doi.org/10.1016/S0010-0277(98)00032-8.

15. Wilson, M. Six views of embodied cognition. Psychon Bull Rev. 2002;9:625-636. doi: https://doi.org/10.3758/BF03196322.

16. Schütz-Bosbach S, Prinz W. Perceptual resonance: action-induced modulation of perception. Trends Cogn Sci. 2007;11:349-355. doi: https://doi.org/10.1016/j.tics.2007.06.005.

17. Faubert J, Sidebottom L. Perceptual-cognitive training of athletes. J Clin Sport Psychol. 2012;6:85-102. doi: https://doi.org/10.1123/jcsp.6.1.85.

18. Bray A, Subanandan A, Isableu B, Ohlmann T, Golding JF, Gresty MA. We are most aware of our place in the world when about to fall. Curr Biol. 2004;14:609-610. doi: https://doi.org/10.1016/j.cub.2004.07.040.

19. Steggemann $Y$, Engbert K, Weigelt M. Selective effects of motor expertise in mental body rotation tasks: comparing object-based and perspective transformations. Brain and Cognition. 2011;76: 97-105. https://doi.org/10.1016/j.bandc.2011.02.013.

20. Zacks JM, Tversky B. Multiple systems for spatial imagery: Transformations of objects and bodies. Spatial Cognit Comput. 2005;5:271-306. doi: https://doi.org/10.1207/s15427633scc0504_1.

21. Kaltner $S$, Jansen P. Sex of human stimulus matters: female and male stimuli are processed differently in mental rotation tasks. Cogn Psychol. 2018;30:854-862. doi: https://doi.org/10.1080/20445911.2018.1530677.

22. Kaltner $S$, Riecke BE, Jansen $P$. Embodied mental rotation: A special link between egocentric transformation and the bodily self. Front Psychol. 2014;5:1-11. doi: https://doi.org/10.3389/fpsyg.2014.00505.

23. Pietsch S, Jansen P. Mental rotation and handedness: differences in object-based and egocentric transformations. Cogn Psychol. 2018;30:511-519. doi: https://doi.org/10.1080/20445911.2018.1483937. 
Brazilian Journal of Motor Behavior

24. Parsons LM. Imagined spatial transformation of one's body. J Exp Psychol Gen. 1987;116:172-191. doi: https://doi.org/10.1037/0096-3445.116.2.172.

25. Prioli AC, Cardozo AS, de Freitas Júnior PB, Barela JA. Task demand effects on postural control in older adults. Hum Movement Sci. 2006;25:435-446. doi: https://doi.org/10.1016/j.humov.2006.03.003.

26. Stins JF, Marmolejo-Ramos F, Hulzinga F, Wenker E, Canal-Brulañd R. Words that move us. The effects of sentences on body sway. Adv Cogn Psychol. 2017;13:156-165. doi: https://doi.org/10.5709/acp-0215-9.

Citation: Budde K, Barela JA, Figueiredo GA, Weigelt M. Mental body rotation with egocentric and object-based transformations in different postures: sitting vs. standing. BJMB. 2020: 14(2): 73-84.

Editors: Dr Fabio Augusto Barbieri - São Paulo State University (UNESP), Bauru, SP, Brazil; Dr Natalia Madalena Rinaldi - Federal University of Espírito Santo (UFES), Vitória, ES, Brazil.

Copyright:@ 2020 Budde, Barela, Figueiredo and Weigelt and BJMB. This is an open-access article distributed under the terms of the Creative Commons Attribution-Non Commercial-No Derivatives 4.0 International License which permits unrestricted use, distribution, and reproduction in any medium, provided the original author and source are credited.

Funding: There was no funding for this study.

Competing interests: The authors have declared that no competing interests exist.

DOl: https://doi.org/10.20338/bjmb.v14i2.165 